\title{
Labyrinthe
}

13 | 2002

Numéro 13

Le débat sur 1948

\section{Une approche des champs de production historique israélien et palestinien}

\section{Philippe Bourmaud}

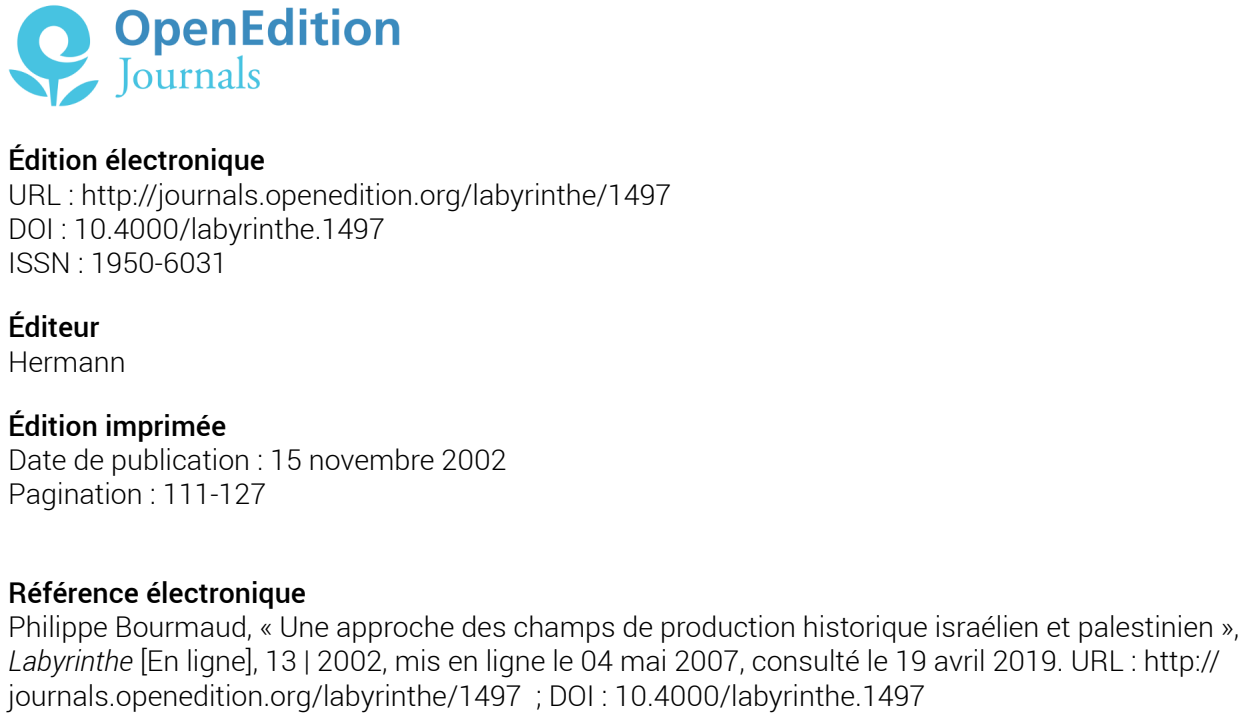

Édition électronique

URL : http://journals.openedition.org/labyrinthe/1497

DOI : $10.4000 /$ labyrinthe. 1497

ISSN : 1950-6031

Éditeur

Hermann

Édition imprimée

Date de publication : 15 novembre 2002

Pagination : 111-127

\section{Référence électronique}

Philippe Bourmaud, «Une approche des champs de production historique israélien et palestinien », Labyrinthe [En ligne], 13 | 2002, mis en ligne le 04 mai 2007, consulté le 19 avril 2019. URL : http:// journals.openedition.org/labyrinthe/1497 ; DOI : 10.4000/labyrinthe.1497

Ce document a été généré automatiquement le 19 avril 2019.

Propriété intellectuelle 


\title{
Le débat sur 1948
}

\section{Une approche des champs de production historique israélien et palestinien}

\author{
Philippe Bourmaud
}

L'article d'Avi Shlaim «Le débat sur $1948^{1}$ », présenté en traduction dans ce numéro, illustre la dimension historiographique du conflit du Proche-Orient. En retraçant la controverse qui a entouré la publication des ouvrages des «nouveaux historiens israéliens ", il laisse voir certaines des rigidités du champ de production historique israélien. Les oppositions, en particulier israéliennes, à la perspective critique de Benny Morris $^{2}$, Ilan Pappé ${ }^{3}$, Tom Segev ${ }^{4}$ ou Avi Shlaim lui-même, illustrent la polarisation très vive des discours scientifiques, qui, selon une perception courante, sont organisés autour de deux récits rivaux et contradictoires de l'histoire contemporaine de la Palestine. Dans cet univers binaire, on présume que chaque camp laisse le point de vue adverse dans le non-dit; on parle des mêmes objets sans paraître parler de la même chose. De fait, des récits très rigides se sont constitués, dans la mesure où les critiques des versions officielles étaient assimilées à l'ennemi et exclues de la discussion académique. À rebours de ces savoirs réifiés, les «nouveaux historiens israéliens » ont fondé leur notoriété sur l'analyse, par une approche documentaire, de ce qu'ils ont eux-mêmes présenté comme des «mythes fondateurs» de leur pays, des références historiques communes empreintes d'imprécision ou d'erreur. Le terme «mythe » peut être employé pour parler des épisodes et des interprétations célèbres repris par l'histoire officielle des deux camps, à condition d'élargir son sens: un ensemble de savoirs transmissibles, représentations sociales, vecteurs d'identification et d'action politique, références investies de contenus émotionnels mobilisables, et données d'un champ de ressources identitaires ${ }^{5}$. Ces sens peuvent exister séparément, mais il nous semble que la fonction de mobilisation et les systèmes de justification produits par les mythes politiques caractérisent leur utilisation tant par les Israéliens que par les Palestiniens. Ces renouvellements historiographiques ont illustré la croyance bien ancrée dans la symétrie des processus: d'aucuns ont 
demandé un travail similaire sur les mythes fondateurs palestiniens. À partir de notre expérience d'enseignant dans une université palestinienne et de chercheur, tant du côté israélien que du côté palestinien ${ }^{6}$, nous aimerions proposer quelques interprétations sur les logiques de production historique, en nous fondant en particulier sur les observations que nous avons pu faire du côté palestinien, que nous connaissons mieux ${ }^{7}$. De fait, nous n'entendons pas mettre rigoureusement en regard les deux camps de la controverse, ce qui supposerait un travail de terrain systématique. Il n'est au reste pas évident que l'idée de symétrie, issue de l'analyse de controverse ${ }^{8}$, soit pertinente pour évoquer le milieu des historiens palestiniens. Un tel postulat conduirait à rendre compte essentiellement des moyens moins importants et de la moindre reconnaissance scientifique des chercheurs palestiniens ; on risquerait en outre de gommer les logiques originales et les difficultés spécifiques de la production historique palestinienne au profit de la stricte mise en regard des récits. L'idée de symétrie ou d'asymétrie, qui scande le débat à la fois historique et politique, touche au contenu des mythes et à l'organisation des champs de production historique. D'une part, il semble que les mythes palestiniens ne tournent pas autour des mêmes aspects que ceux qu'examinent les nouveaux historiens israéliens dans leur œuvre de démystification. Les mythes de l'un et l'autre camp reposent sur des ressorts argumentatifs et émotionnels différents, et ne sont pas susceptibles d'un même travail critique ou herméneutique. D'autre part, les champs de production scientifique, où s'élaborent récits et renouvellements historiographiques, ne semblent pas réductibles à un jeu de miroirs. Les contraintes de la production historique illustrent des usages sociaux très construits de l'historiographie. C'est du moins ce que suggère l'analyse de la production historiographique concernant la Palestine $\mathrm{du} \mathrm{XIX}^{\mathrm{e}}$ siècle aux années 1948-1952.

Nation, État et histoire officielle

2 Les versions officielles de l'un et l'autre camp reflètent des expériences très différentes: celles de l'État hébreu définissent, à travers quelques épisodes, des références collectives qui illustrent l'ethos sioniste. Ils constituent un appareil de légitimation nationale, relayé par les institutions éducatives et militaires. Dans ce contexte, la «nouvelle histoire d'Israël », écrite par des Israéliens juifs, peut être analysée comme une critique de la construction nationale et un gage de la solidité de l'État.

3 L'analyse des mythes historiques est, en revanche, plus délicate dans le cas palestinien. L'absence d'État ne permet pas d'étudier un processus de construction d'une histoire étatique. Les historiens palestiniens ont dû travailler dans des conditions très variables, qui ont orienté leur production. Les travaux des années 1930 au début des années 1950 prennent généralement la forme de la chronique, et visent en particulier à défendre, au moyen de l'histoire, la cause arabe palestinienne contemporaine ${ }^{9}$. C'est ce que l'on retrouve dans les ouvrages de Georges Antonius ${ }^{10}$ et de "Ârif al-'Ârif ${ }^{11}$. Après la Nakbah est apparue une histoire palestinienne aux courants variés, qui ont eu en commun leur adhésion au mouvement national palestinien et le souci de comprendre la condition palestinienne. La première tâche a alors été, à la fin des années 1950, de réfuter l'historiographie israélienne du conflit et de la guerre de 1948 par des moyens académiques. Walid Khalidi s'est en particulier attaché à invalider l'argument principal des dirigeants israéliens ; à savoir, que les Arabes palestiniens avaient fui leur domicile de leur plein gré, à l'appel des radios arabes qui leur auraient promis un retour prochain après la défaite sioniste. Les scripts des émissions de radio arabe de 1948 ne laissent nulle part apparaitre de tels ordres ${ }^{12}$. En outre, Khalidi a exposé un ordre stratégique adopté en 
mars 1948, le plan Dalet, qui peut être interprété comme un plan impliquant l'expulsion des Arabes palestiniens ${ }^{13}$. Dans les années 1960, une seconde étape a été de faire un inventaire documenté et chiffré de la dépossession: Sami Hadawi, qui travaillait pour la commission de l'ONU chargée de réfléchir à la compensation des réfugiés, a écrit dans une veine marxiste l'histoire et la liste de ces pertes ${ }^{14}$. Les travaux des années 1960 et 1970 sont à comprendre dans le contexte de la « révolution palestinienne » entamée par l'OLP, qui visait à libérer la terre et la société palestiniennes, sous l'influence des mouvements anticolonialistes de gauche. Ils ont notamment cherché à analyser l'influence de la division "féodale» de la société arabe palestinienne traditionnelle, comme un des facteurs de la Nakbah ${ }^{15}$. L'échec de la stratégie au début des années 1980 s'est traduit parmi les historiens par un souci de préserver les traces de la Palestine ancienne, au moyen d'une collecte des traditions et souvenirs oraux constituant l'expérience palestinienne, et d'écrire celle-ci conformément aux normes académiques ${ }^{16}$. Dans les années 1990, les efforts de l'Institut d'études palestiniennes pour favoriser la reconnaissance scientifique des historiens de la Palestine ont abouti à la parution d'ouvrages qui donnent un panorama plus varié. Ces travaux ont en commun de procéder à un travail de décryptage des présupposés idéologiques des historiens des générations précédentes ${ }^{17}$, et à la reconstruction d'espaces palestiniens différenciés ${ }^{18}$. En dépit de leurs différences d'approches, ces historiens estiment souvent avoir à défendre l'existence d'une communauté palestinienne distincte, puisque les buts nationaux sont encore à réaliser ${ }^{19}$. Au reste, l'investissement mythique de l'histoire constitue un jeu de références éclatées, à côté des discours de l'histoire professionnelle: activités de lutte, culture populaire, publications partisanes, etc. contribuent à construire des mythes.

4 Comme le récit sioniste, le récit palestinien s'inscrit dans la longue durée, et les anthropologues palestiniens s'efforcent de rattacher les coutumes palestiniennes aux découvertes sur les pratiques des populations du Croissant fertile dans la Haute Antiquité. Les Cananéens et les Philistins sont une des strates des peuplements que la mémoire sélective a rattachées à l'identité revendiquée par les Palestiniens ${ }^{20}$. La conception archéologique du droit d'ancienneté est l'aspect le plus directement lié à une stratégie de défense pied à pied contre l'argumentaire sioniste. Ce mythe explique, par exemple, le désir de fêter le cinq millième anniversaire de Jérusalem, en se fondant sur l'archéologie des premiers établissements humains, en contrepoint des fêtes des trois mille ans de la ville de David, en $1998^{21}$. Il va de soi que l'enjeu est alors purement politique, savoir : la revendication sur Jérusalem, et en particulier sur la Vieille Ville et le quartier de Silwad, où se trouvent les ruines les plus anciennes.

5 Les Palestiniens perçoivent leur histoire récente comme celle d'une société en lutte, héritière des guerres de l'Islam. Les résistances des habitants de la Palestine fondent la continuité du mouvement national palestinien, qui s'insère dans une plus vaste histoire du monde musulman, et s'inspire d'hommes, de lieux et d'événements marquants, qui préfigurent la lutte palestinienne contemporaine. Le discours sur la lutte est mis en valeur au moyen de matériaux très divers : anecdotes et contes, chansons, et toutes les formes de la culture populaire ${ }^{22}$. C'est un vecteur de transmission qui entoure aussi bien les Croisades ou la révolte arabe de 1936-1939 que la première Intifada. Les endroits associés à la mémoire de Saladin ont été l'enjeu de luttes politiques ; ainsi du Nabi Mousa, lieu saint abritant le tombeau de Moïse, accueillant un pèlerinage fondé au XIIe siècle sur ordre de Saladin, selon la tradition. Ce dernier lieu fut le grand lieu de mobilisation politique arabe palestinienne sous le mandat. Les hommes des trois grandes villes de 
l'intérieur (Jérusalem, Hébron et Naplouse) y renchérissaient de patriotisme local et national, bientôt encadrés par certains des partis rivaux qui animaient le mouvement national palestinien sous le mandat britannique ${ }^{23}$. Le pèlerinage devint dans les années 1920 un enjeu de démonstration de force politique pour les partis de toute la Palestine, et des hommes politiques chrétiens, tel Khalîl al-Sakâkînî, s'agrégèrent au cortège pour manifester leur esprit nationaliste ${ }^{24}$. Le discours mythique s'approprie l'histoire de l'Islam dans la longue durée et l'histoire locale.

Le récit du conflit entre Arabes et sionistes en Palestine est cependant borné dans une période de temps bien plus courte, d'environ cent vingt ans : c'est, pour les Palestiniens, la période de la dépossession. Si la version officielle israélienne insiste sur la période suivant l'arrivée des premiers sionistes en Palestine en 1882, le récit palestinien insiste sur les transformations préalables de la Palestine, au milieu desquelles le conflit s'est mis en place. La modernisation et l'occidentalisation de l'Empire ottoman dans les années 1850 constituent le contexte général dans lequel la lente expropriation des paysans arabes palestiniens au profit des colonies agricoles sionistes a été possible. La source des malheurs des Palestiniens est souvent rapportée à la loi ottomane de 1858, qui prévoyait que les terres d'État pouvaient désormais être vendues en pleine propriété privée : cette loi aurait ouvert la porte à la colonisation agricole sioniste et à l'éviction des Palestiniens de leur terre. Les propriétaires absentéistes de Jaffa, Haïfa et Beyrouth sont accusés d'avoir tiré profit de cette mesure en acquérant la propriété des terres, en rationalisant les cultures au mépris des droits traditionnels des paysans, et en revendant cher les exploitations aux institutions sionistes, souvent à l'insu des paysans. Dans la mesure où les colonies agricoles sionistes excluaient le travail arabe, de nombreux paysans palestiniens se sont trouvés évincés de leurs terres. Pourtant, selon Bichara Doumani, la transformation des terres d'État en propriété privée était déjà en cours depuis les années 1820 ou $1830^{25}$ : la loi ottomane, et avec elle la politique de réforme encouragée par l'Occident dans les territoires du sultan, n'est donc pas l'origine exclusive des expropriations de paysans palestiniens. Le mythe de la loi de 1858 s'explique par les circonstances dans lesquelles l'histoire de la Palestine s'est élaborée à partir des années 1930-1940. À cette époque, la paysannerie arabe palestinienne traversait une crise profonde, liée tant aux acquisitions de terres par les institutions sionistes qu'aux arriérés de dettes des fermiers à l'égard des grands propriétaires. La révolte arabe de 1936-1939 était dominée par la paysannerie, et se tourna à la fois contre les Britanniques et le mouvement sioniste, et contre le pouvoir des grands propriétaires vivant dans les grandes villes. C'est à partir de là que le mouvement national palestinien a été associé au sort de la paysannerie, et que l'histoire de la Palestine a été construite comme l'histoire des malheurs des paysans palestiniens.

7 Les différentes temporalités $\mathrm{du}$ récit palestinien convergent vers une période extrêmement brève, qui fonde à la fois le malheur des Palestiniens et leur identité actuelle, irréductible à l'expérience d'aucun de leurs voisins. Le point focal du récit palestinien est la Nakbah, l'expulsion d'environ $70 \%$ de la population arabe de Palestine en quelques mois de l'année 1948. L'histoire de l'exode, phénomène macroscopique à l'échelle de la Palestine, est construite sur l'agrégat des histoires individuelles, notamment le souvenir de la terre possédée. Les histoires de la Nakbah de 1948 se répètent et se conjuguent pour former de nouveaux mythes qui investissent la culture matérielle des réfugiés : ainsi en est-il des clés de la demeure abandonnée, matière à 
manifestation; de même de l'oreiller que de nombreux réfugiés rapportent avoir emporté, dans leur panique, en lieu et place du couffin du dernier-néér.

Le temps d'après la Nakbah est celui de l'exil ou de l'occupation. Le mouvement national palestinien s'y reconstruit par rapport à des gouvernements locaux hostiles ou embarrassés. C'est à partir de ce moment-là que prend forme le mythe de la trahison des Arabes, qui se reproduit à l'infini et se construit perpétuellement à partir de 1948: la défaite face à Israël est vue comme le résultat des menées souterraines de l'émir Abdallah de Transjordanie, l'éviction de l'OLP hors de Jordanie en septembre 1970 est encore attribuée au soutien d'Israël au roi Hussein contre les Palestiniens, l'éviction de l'OLP hors du Liban est imputée aux Libanais, et aux chrétiens de ce pays en particulier, et ainsi de suite. Sous les sentiments ambigus de solidarité très émotionnelle et d'impatience mutuelle, les relations entre Palestiniens et États arabes ont constitué un des nœuds de réflexion de l'historiographie nationaliste arabe palestinienne qui a dominé jusqu'aux années 1980. À cette perspective, une historiographie d'inspiration islamique ${ }^{27}$, à partir de la révolution iranienne, a opposé une autre solidarité, celle de l'ummah, la communauté des musulmans. Chacun de ces réseaux de solidarité suscite des réactions émotionnelles, traduites par des proclamations enflammées tant aux congrès de la Ligue arabe qu'à ceux de l'Organisation de la conférence islamique, mais ces solidarités sont des constructions intellectuelles, dans une région où le jeu politique est extrêmement tendu. À rebours des conceptions essentialistes des orientalistes du siècle passé, il parait minimal d'évoquer une pluralité des peuples arabes ${ }^{28}$, et des intérêts divergents de pays à pays; ce qui ne diminue en rien le sentiment d'abandon. Il s'ensuit que de nombreux Palestiniens ont le sentiment d'être des pions dans un jeu abstrait de puissances régionales ou dans un vaste complot.

$9 \quad$ La liste n'est pas exhaustive. Avec le temps, cette mythologie est devenue clairement un enjeu politique. De façon caractéristique, les débats qui ont suivi l'expulsion de l'OLP du Liban après le très dur siège de Beyrouth par l'armée israélienne à l'été de 1982 ont tourné autour de l'utilisation de cet épisode pour la propagande palestinienne. Yasser Arafat voulait utiliser la résistance acharnée des Palestiniens pour glorifier la détermination $d u$ mouvement national palestinien, mobiliser une nouvelle fois les Palestiniens de l'intérieur et du dehors, et consolider sa direction contestée. Une partie de la direction palestinienne, par la voix du représentant de l'OLP au Liban, Shafiq alHout, a contesté la présentation de la bataille comme un nouvel épisode de la glorieuse et vaine - résistance palestinienne ${ }^{29}$. L'expulsion de Jordanie en 1970, puis du Liban en 1982, privait l'OLP de toute frontière avec Israël, et donc de toute stratégie militaire. Un nouvel appel à la mobilisation risquait de montrer la direction politique palestinienne isolée, coupée de la réalité et privée de perspective stratégique. Le discours historique, mythique ou non, est soumis à une double finalité: proposer aux Palestiniens un témoignage de leur passé et une ouverture sur l'avenir, et défendre la cause palestinienne aux yeux du monde.

Champs de production historique et représentations sociales

10 Le conflit, qui façonne les mythes nationaux tant israéliens que palestiniens, organise les champs de production historique tant du point de vue des méthodes de l'historien que de celui de la réception des travaux. L'option positiviste et documentaire des nouveaux historiens israéliens traduit une volonté de désamorcer le débat en se retranchant derrière la source textuelle ${ }^{30}$. L'étape suivante, que représente l'utilisation des sources orales sur certains épisodes critiques de l'histoire nationale israélienne, est pour partie 
l'exact prolongement des méthodologies rodées en Israël même pour l'histoire orale d'événements traumatiques, à commencer par la Shoah ${ }^{31}$, en dépit de fortes réticences mentales et méthodologiques à l'égard de «l'imagination $\operatorname{arabe}^{32}$ » chez certains historiens.

11 Les mêmes méthodologies se retrouvent dans la production palestinienne, pour des motifs autres. L'accumulation et la critique des sources orales est le moyen de préserver une réalité évanescente avant que les témoins ne soient tous morts, et alors qu'une bonne partie des paysages et des sources locales arabes a été détruite. Le recours à l'oralité est évident aux yeux d'une population palestinienne dont les réfugiés font un usage intensif de la mémoire: les habitants des camps se distinguent par leurs villages d'origine, connaissent par une description précise l'aspect et le site de leur ancienne maison. Cela explique le « Projet des villages palestiniens disparus », de l'université de Bir Zeit. De 1984 au début des années 1990, les chercheurs de ce centre ont recueilli des témoignages oraux, dispersés dans le monde arabe, sur une quinzaine de villages disparus après l'expulsion de $1948^{33}$. Le souci documentaire se rattache également à un souci de conservation des traces et des preuves, alors même que le peuple palestinien manque d'institutions étatiques de conservation.

12 Le principal effort de conservation a été celui des Jordaniens, qui ont microfilmé entre 1949 et 1967 les manuscrits des archives des fondations charitables de mainmortes (awqâf ) et des tribunaux religieux (mahâkim sharîiyyah), ainsi que les collections des journaux palestiniens. Le tout est conservé au département des manuscrits de l'université jordanienne, université dont la réputation a, du reste, largement bénéficié de l'accueil des chercheurs palestiniens. Leur consultation suppose, pour les Palestiniens, l'autorisation de passer les frontières, qui n'est pas toujours accordée par l'État d'Israël, par exemple pour raison de militantisme nationaliste palestinien. Ces archives sont essentielles tant pour les historiens de la Palestine que pour tout travail sur les structures de propriété dans les Territoires palestiniens. Elles sont également accessibles à la bibliothèque du Haram al-Sharif, tenue par l'administration religieuse de l'Esplanade des mosquées de Jérusalem, mais seuls les Palestiniens résidents de Jérusalem ont droit de fréquenter les lieux. Pour les Palestiniens de Cisjordanie, qui n'ont plus le droit d'entrer à Jérusalem depuis le début de la seconde Intifada, d'autres microfilms sont présents dans les ministères palestiniens responsables du patrimoine palestinien et des tribunaux religieux, dans les villages d'Abu Dîs et d'Al-'Izzariyyeh, banlieues en dehors des limites municipales de Jérusalem, bien que le découpage récent de la Cisjordanie ait aussi rendu ce voyage compliqué. Pour les universitaires gaziotes, enfin, de telles recherches sont désormais exclues, depuis l'arrêt de la délivrance des laissez-passer de Gaza en Cisjordanie en septembre 2000. L'existence de ministères dotés d'archives a une signification de plus en plus trompeuse, faute de continuité territoriale. L'exercice du métier d'historien est donc particulièrement compliqué pour les Palestiniens, et la valorisation nationaliste de l'histoire ne doit pas conduire à surestimer la position sociale des historiens et la diffusion de leurs travaux dans la population palestinienne. En comparaison de la controverse qui a éclaté dans le public israélien après la publication des travaux des "nouveaux historiens israéliens ", ces ouvrages ont été accueillis avec faveur par les universitaires palestiniens. Leurs auteurs semblaient donner raison aux Palestiniens; les critiques émises par les historiens palestiniens ${ }^{34}$ concernaient essentiellement les conclusions des nouveaux historiens. Benny Morris, en particulier, était accusé de minimiser la portée de ses découvertes. 

spécialistes israéliens mais non au-delà. Ilan Pappé le note ironiquement au sujet du grand historien palestinien Walid Khalidi. Les travaux de celui-ci sur l'expulsion de 1948 et sur les villages détruits après la Nakbah n'étaient généralement pas pris au sérieux dans les milieux académiques israéliens; ils n'ont trouvé un début de reconnaissance qu'avec «l'Affaire Teddy Katz », et de façon paradoxale. En quelques mots, Teddy Katz, étudiant à l'université de Haïfa a présenté en 2000 un mémoire démontrant le massacre d'une partie de la population du village arabe palestinien de Tantoura en mai 1948, au moyen de sources orales israéliennes et palestiniennes. Corrigé par des historiens réputés tels que Meir Pa'il et Ilan Pappé, le mémoire a été applaudi avant que certains des témoins israéliens ne contestent l'utilisation de leur témoignage et n'engagent une procédure judiciaire. Celle-ci s'est achevée sur une rétractation de Teddy Katz, dans des circonstances discutées. Lors de la controverse qui a éclaté en Israël, des historiens israéliens ont soudain trouvé du mérite à Walid Khalidi, qui n'avait justement pas mentionner ce massacre dans ses ouvrages ${ }^{35}$. Au sein de la société civile et des médias, l'accueil réservé aux travaux historiques est significatif. Côté israélien, les séries d'articles occasionnées par la publication des travaux des nouveaux historiens puis, sur un mode quasi unanimement critique, par la thèse de Teddy Katz, ont montré à la fois qu'il existe un intérêt du public pour les renouvellements historiographiques, et que les réévaluations historiques du sionisme affectent des aspects essentiels de la conscience nationale israélienne. À l'extérieur, ces enjeux prennent une autre tournure, comme on l'a vu récemment avec l'Affaire Pappé dans les journaux occidentaux : c'est désormais l'indépendance scientifique des milieux académiques israéliens qui est mise en cause à travers le prisme de l'historiographie ${ }^{36}$. Côté palestinien, il faut distinguer la valorisation de la recherche historique et le statut de l'historien. Le statut de l'histoire comme discipline universitaire, et par ricochet celui de l'historien, est dévalué. L'historien bénéficie du prestige du diplôme ${ }^{37}$, mais la matière en elle-même est nettement moins valorisée que les matières scientifiques, qui attirent les meilleurs bacheliers. À l'inverse, les centres de recherche historique ont réussi à drainer des fonds importants de la diaspora après les accords d'Oslo. Cela étant posé, le poids de la recherche historique palestinienne est très faible, ce qui découle largement des formes de contrôle qu'instituait l'occupation directe. Les bibliothèques privées palestiniennes ont longtemps mené une politique de secret par crainte de l'administration israélienne. Les centres disposant d'archives restaient discrets, et leurs fonds étaient souvent réputés pour leur manque de classement. Ainsi, la bibliothèque Ansariyyeh, en face de l'hôtel Saint-Georges à Jérusalem, est cachée dans un sous-sol où l'on accède par l'arrière d'une maison. Comme d'autres bibliothèques de Jérusalem, elle a été créée par une grande famille, richement dotée, mais ne s'autorise aucune publicité. Le thé est servi sur des tables parfois jonchées de vieux manuscrits, et le chercheur doit se fier à la connaissance très approfondie que le bibliothécaire a de son fonds. L'État d'Israël exerçait en outre un contrôle intellectuel, qui passait par la distribution de manuels scolaires égyptiens et jordaniens expurgés ${ }^{38}$ et par l'octroi ou non du titre d'université aux collèges académiques palestiniens. La discussion qui a abouti à l'élévation du collège An-Najah de Naplouse au rang d'université en 1978 tournait autour de la réputation de nationalisme de cette institution, et, corrélativement, des programmes d'histoire. Aujourd'hui, le cours d'histoire commun de la faculté des arts de cette même université s'intitule "La cause palestinienne » et vise en particulier à assimiler le contenu d'un manuel éponyme. L'intitulé du cours affirme clairement la portée politique du type d'histoire qui est enseigné. Il s'en faut de beaucoup, cependant, 
que l'on puisse parler d'endoctrinement, car ces cours sont le théâtre d'âpres disputes ; le résultat de cet enseignement est surtout que les étudiants ont une connaissance de certains aspects précis, mis en relief en cours ou dans le manuel, mais assortie de larges lacunes.

Tant l'État d'Israël que l'Autorité palestinienne sont restés très en retrait des découvertes des dernières années. Le recours à la justice et la constitution d'une vérité de la chose jugée, dans l'Affaire Katz, ne doit pas cacher cependant le fait que la légitimité historiographique vient pour bonne part de l'étranger. À ce compte, il est clair que les milieux académiques israéliens ont un avantage de formation et de notoriété, cependant que les centres de recherche palestiniens en sont à se constituer une reconnaissance internationale ; et celle-ci est déjà très inégale, qui va d'abord à l'université de Bir Zeit et aux milieux intellectuels de Ramallah et de Jérusalem-Est. La présentation des travaux palestiniens est encore soumise à un principe intériorisé de symétrie de participation, un jeu de balance dans les colloques: avant l'éclatement de la seconde Intifada, les organisateurs des colloques de l'université de Bir Zeit s'efforçaient de réunir des chercheurs des deux camps ${ }^{39}$. Le principe de la confrontation des points de vue révèle cependant des positions bien campées, sans réelle discussion. C'était particulièrement clair lors du colloque du palais du Luxembourg de mai 1998, organisé par Le Monde diplomatique et la Revue d'études palestiniennes, qui réunissait historiens palestiniens et «nouveaux historiens israéliens »: la discussion s'est focalisée autour des différends entre les uns et les autres, sans modification des positions ${ }^{40}$.

Le conflit imprègne le débat scientifique, et par le biais de stratégies argumentatives ambiguës, le discours politique pénètre les bibliographies des sciences humaines, politiques et sociales, au moyen de constructions taxinomiques et d'exigences contradictoires. On retrouve ici, sorties du contexte politique, les critiques pour " unilatéralisme ", ou pour utilisation abusive d'auteurs "marginaux» ou "politisés ». Les bibliothèques que nous avons visitées sont très hétérogènes quant au public, à la quantité de livres disponibles et aux moyens financiers pour l'achat des nouveautés. Surtout, elles ne nous disent rien de l'utilisation des ouvrages dans les listes de lecture des étudiants. Celles-ci dépendent de l'orientation des départements. Du côté israélien, les intitulés des centres de recherche et des sections d'enseignement ont souvent un fort contenu politique. Les départements du Moyen-Orient ont longtemps constitué la pépinière des services de sécurité de l'État d'Israël, mais ils sont en même temps souvent plus marqués à gauche que les départements d'histoire, et surtout que les départements d'étude de la Terre d'Israël. Cette dernière appellation recouvre un concept très politisé de l'espace revendiqué par Israël, déterminé en fonction de références bibliques et de la situation présente ${ }^{41}$.

16 Du côté palestinien, la recherche historique est le fait des départements d'histoire et d'archéologie. Les spécialistes des études arabes, qui s'occupent généralement de l'ensemble des sources de langue arabe, font également souvent œuvre d'historiens. Ces divisions ne sont pas politisées en elles-mêmes, mais selon les universités la marge d'expression des chercheurs est plus ou moins limitée : les publications scientifiques de certaines universités palestiniennes n'accueillent pas de points de vue hétérodoxes. Les universités qui se dégagent du lot, Bir Zeit au premier rang, sont précisément celles qui ont acquis une réputation d'autonomie politique.

17 La population étudiante des universités que nous connaissons varie fortement, entre l'écrasante majorité juive israélienne de l'université hébraïque, le côtoiement d'Israéliens 
juifs et Bédouins à Beer Sheva, la bourgeoisie palestinienne de Bir Zeit, le milieu plus rural de l'université An-Najah. Les deux universités israéliennes considérées possèdent une quantité d'ouvrages sur l'histoire de la Palestine et du conflit israélo-arabe, avec dans les deux cas une séparation géographique entre, d'un côté, l'histoire du mouvement sioniste et de l'état d'Israël, placée dans l'histoire juive et, de l'autre, l'histoire de la Palestine et des Palestiniens, adjointe ou mêlée à l'histoire des pays arabes. La bibliographie palestinienne, incomplète, n'est pas rejetée dans le néant. La dichotomie bibliographique, qui illustre l'idée d'une dualité d'objets, et notamment d'un développement social « à deux vitesses ${ }^{42}$ » des sociétés juives et arabes en Palestine, ne se retrouve pas dans les bibliothèques académiques palestiniennes, où l'insertion dans le contexte géographique prime. Le choix de livres y est cependant également binational, même si certains thèmes privilégiés, tels que celui des réfugiés, comptent principalement des titres arabes - c'est d'ailleurs une réalité de production plutôt qu'une politique d'achat. Du côté palestinien, la richesse bibliographique est plus nette à l'université de Bir Zeit, centre de recherche financé par la diaspora et de réputation internationale.

L'interprétation des récits historiques est influencée par des partis pris théoriques, qui oscillent entre l'objectivisme et le post-modernisme. C'est à la confluence des milieux intellectuels palestiniens et de l'extrême gauche israélienne, dans les rares espaces de dialogue intellectuel, que l'on peut trouver les meilleures illustrations de ces balancements herméneutiques. Un bon exemple en a été la réunion d'un groupe de dialogue académique israélo-palestinien qui s'est tenue à Al-Ram en mai 2001. On y trouvait des intellectuels palestiniens et israéliens, des activistes israéliens et un parterre d'activistes, chercheurs et journalistes occidentaux.

L'un des enjeux du débat était la discussion de « 80 thèses pour la refondation du camp de la paix " publiées par l'activiste et journaliste d'extrême gauche Uri Avnery ${ }^{43}$. L'auteur utilisait, de façon plutôt opportuniste, l'appareil post-moderniste d'analyse impartiale des récits historiques concurrents, pour fonder la légitimité des deux mouvements de libération nationale sioniste et palestinien et désamorcer la rancœur des uns et des autres. Il proposait des réponses programmatiques, qui permettraient à la gauche pacifiste israélienne de se reconstituer sur des bases moins opaques que celles d'Oslo. Ce ne fut pas le contenu historique de ces propositions qui fut l'objet principal des critiques, dans la mesure où il était perçu unanimement comme lénifiant et inacceptable pour n'importe quel Palestinien instruit. Le débat portait davantage sur l'inspiration théorique du texte, qui reposait sur un équilibre entre les deux points de vue. La démarche d'Avnery se heurtait au sentiment général des participants que les thèses faisaient l'impasse sur les responsabilités dans le conflit. L'un des activistes israéliens émettait l'idée que le texte eût été grosso modo acceptable si l'on avait brisé le parallèle entre les deux camps en rendant compte $\mathrm{du}$ récit palestinien et $\mathrm{du}$ récit colonial israélien. L'adjonction $\mathrm{du}$ qualificatif « colonial » met l'accent sur le projet global d'un des deux camps, et permet de rompre d'emblée la symétrie en raison de l'opprobre qui enveloppe les intentions coloniales depuis les années 1960. Dans ce contexte intellectuel, le sionisme est interprété comme un mouvement nationaliste, mais ne peut être mis en regard d'un mouvement de libération nationale comme celui des Palestiniens, car le colonialisme et la libération sont antithétiques. Du côté palestinien, la possibilité de ce relativisme narratif était contestée au nom de la dénonciation de la domination objective. Les moyens très réduits des Palestiniens, en face de la puissance israélienne, et leur demande de justice n'autorisent aucune symétrie, car ils se conçoivent comme les victimes du conflit. Le relativisme ne 
séduit pas les Palestiniens, qui attendent encore que leur tragédie prenne fin, et qu'une réalisation de leurs aspirations nationales leur permette de vivre dans la stabilité et de prendre des distances à l'égard de leurs revendications générales et de leurs stratégies.

L'asymétrie des champs de production historique israélien et palestinien et de la reconnaissance des travaux des chercheurs de l'un et l'autre camp découle de la construction du conflit politique et de ses références mythiques de part et d'autre, mais également du soutien ou non d'un État constitué à l'écriture de l'histoire et à la diffusion des savoirs. Dans le cas israélien, la place de l'État se révèle un obstacle à l'historiographie critique, cependant que l'absence d'État pèse sur la possibilité d'une profession historienne palestinienne. Il est clair que la seconde Intifada, en marginalisant les analyses critiques $\mathrm{du}$ consensus national israélien et en rendant la recherche matériellement difficile pour les chercheurs palestiniens, est un élément de dissuasion puissant. De plus, le recours au jugement des centres de recherche à l'étranger met l'accent sur les logiques de conflit au sein de la profession historienne. Cela étant posé, les champs de production historique considérés sont marqués par des processus d'étiquetage plus complexes (politique, disciplinaire, etc.) qui pèsent individuellement et fortement sur la réception des travaux.

\section{NOTES}

1. «The Debate about 1948 », dans International Journal of Middle East Studies, vol. 27, n 3, août 1995, pp. 287-304.

2. The Birth of the Palestinian Refugee Problem 1947-1949, Cambridge, Cambridge University Press, 1989.

3. La Guerre de 1948 en Palestine. Aux origines du conflit israélo-arabe, Paris, La Fabrique, 2000.

4. Les Premiers Israéliens (1949-1951), Paris, Calmann-Lévy, 1998.

5. Voir Raoul Girardet, Mythes et Mythologies politiques, Paris, Seuil, 1984.

6. L'auteur de cet article, doctorant en histoire à l'université de Provence et à l'Iremam, a été d'octobre 2000 à septembre 2001 lecteur de français à l'université nationale An-Najah de Naplouse ; il est retourné poursuivre ses recherches en Israël et en Palestine en décembre 2001 et janvier 2002. Les observations qui suivent sont la mise en perspective de cette expérience. Il tient à remercier en particulier Vincent Romani, Aude Signolles et Jihane Sfeir-Khayyat pour leurs conseils et leurs remarques.

7. L'article d'Avi Shlaim traduit dans ce même numéro donne pour le reste un exemple du fonctionnement du champ de production historique israélien, et des interactions entre les historiens acteurs de la production historique autour de 1948 dans le cadre d'une controverse.

8. Sur la question de l'impartialité dans l'analyse de controverse, voir notamment Bruno Latour, Steve Woolgar \& Jonas Salk, Laboratory Life, Princeton, Princeton University Press, 1986 ; et Malcom Ashmore \& Steve Woolgar, The Reflexive Thesis : writing sociology of scientific knowledge, Chicago, University of Chicago Press, 1989. 
9. Elias Sanbar, « Hors du lieu, hors du temps. Pratiques palestiniennes de l'histoire », dans François Hartog et Jacques Revel (dir.), Les Usages politiques du passé, coll. « Enquête ", Paris, Éditions de l'École des hautes études en sciences sociales, 2001, pp. 117-125.

10. The Arab Awakening, Londres, Hamish Hamilton, 1938.

11. Al-Nakba, 1947-1952 (Le Désastre, 1947-1952), 6 vol., Beyrouth, al-Maktaba al-'Asriya, 1956-1960.

12. Walid Khalidi, « What Made the Palestinians Leave? ", dans Middle East Forum, juillet 1959, pp. 21-24.

13. Walid Khalidi, «Plan Dalet : The Zionist Masterplan for the Conquest of Palestine, 1948 ", dans Middle East Forum, novembre 1961, pp. 22-28. C'est autour de ce plan que s'est concentrée, trente ans plus tard, la controverse entre Benny Morris et les historiens palestiniens. Selon le premier, ce texte n'est pas un ordre d'expulsion, et l'éviction des Palestiniens a été le résultat des opérations militaires au coup par coup (voir Benny Morris, The Birth of the Palestinian Refugee Problem, 1947-1949, Cambridge, Cambridge University Press, 1987, p. 286). Pour les seconds, ce document est la preuve que le départ forcé des Arabes palestiniens était planifié (voir Nûr Masalha, « Le concept de « transfert » dans la doctrine et dans la pratique du mouvement sioniste », dans Elias Sanbar \& Farouk Mardam-Bey (dir.), Le Droit au retour, coll. Sindbad, Paris, Actes Sud, 2002, pp. 29-51). Le débat s'installe entre les interprétations intentionnaliste et pragmatique de la Nakbah.

14. Palestinian Rights and Losses in 1948. A Comprehensive Study, Londres, Saqi Books, 1988. La première édition date des années 1960.

15. Cette historiographie a été soutenue par l'OLP et ses différentes composantes : voir l'ouvrage de Naji 'Allouch, Al-Muqâwama al-'arabiyya fi Filastîn (La Résistance arabe en Palestine), Beyrouth, Centre de recherche de l'OLP, 1974 ; et le livre de l'écrivain et porteparole du FPLP, Ghassan Kanafânî, Thawrat 1936/1939 fî Filastîn - Khalifyyât wa tafassîl wa tahlîl (La Révolte de 1936/1939 en Palestine), Beyrouth, Éditions du FPLP, 1974.

16. Sharif Kanaana, Still on Vacation!, Jérusalem, SHAML, 2000 ; « Palestinian National Identity and the Palestinian Folklore Movement » dans Sharif Kanaana, (dir.) Folk Heritage of Palestine, Taybeh, Research Center for Arab Heritage, 1994, pp. 1-15.

17. 'Âdil Mannấ, Târih Filastîn fî awâhir al-'ahd al-'utmânî 1800-1918 (qirâ'a jadîda) [L'Histoire de la Palestine à la fin de l'époque ottomane 1800-1918 (une relecture)], Beyrouth, Institut d'études palestiniennes, 1999.

18. Bichara Doumani, Rediscovering Palestine. Peasants and Merchants of Jabal Nablus in the Nineteenth Century, Berkeley, University of California Press, 1995.

19. Cela étant, la recherche universitaire palestinienne, depuis 1993, tend à se dégager d'une perspective de « défense et illustration de la Palestine », voir Elias Sanbar, art. cit., pp. 123-124.

20. En cela, l'identité « arabe cananéenne » des Palestiniens diffère de l'alternative entre identité phénicienne et identité arabe qui a longtemps marqué le débat politique et historique libanais.

21. Meron Benvenisti, City of Stone. The Hidden History of Jerusalem, Berkeley, L. A \& Londres, University of California Press, 1996, pp. 1-4.

22. Sharif Kanaana, « Palestinian National Identity and the Palestinian Folklore Movement », dans Folk Heritage of Palestine, Taiybeh, Research Center for Arab Heritage, 1994, pp. 1-15. 
23. Tom Segev, One Palestine, Complete. Jews and Arabs under the British Mandate (traduit en français sous le titre C'était en Palestine au temps des coquelicots, Paris, Liana Levi, 2000), Londres, Little Brown \& Co, 2001, pp. 126-143.

24. Emma Aubin-Boltanski, « Moïse ou Salâh ad-Dîn : pèlerinage religieux/pèlerinage politique en Palestine » dans La Palestine en transition. Crise du projet national et construction de l'État, collection « Les Annales de l'autre Islam », Paris, Publication de l'Erism, Inalco, 2001, pp. 373-397.

25. Bichara Doumani, op. cit., pp. 155-164.

26. On pourra trouver une illustration audiovisuelle du thème symbolique des clés dans le film de Maï Masri, Rêves d'exil (2000). La réalisatrice montre en particulier l'organisation d'une manifestation de clés en carton par une association de jeunes réfugiés du camp de Chatila, et l'entretien de ces jeunes avec un homme qui a vécu la Nakbah, et qui insiste sur le caractère mémorialiste et non réaliste de cet objet.

27. 'Âdil Manna', op. cit., pp. 1-4.

28. Albert Hourani, Histoire des peuples arabes, Paris, Seuil, 1989.

29. Rashid Khalidi, Palestinian Identity, New York, Columbia University Press, 1997, pp. 199-200.

30. Pour une analyse de la sociologie du débat entre historiens israéliens, voir Uri Ram, « Mémoire et identité : sociologie du débat des historiens en Israël », dans Florence Heymann \& Michel Abitbol, L'Historiographie israélienne aujourdhui, coll. « Mélanges du CRJF », Paris, Éditions du CNRS, 1998, pp. 197-243.

31. Ilan Pappé, «L'affaire Tantoura en Israël », dans la Revue d'études palestiniennes, 28, été 2001, pp. 55-56.

32. Voir par exemple la discussion par Norman Finkelstein de la permanence de cette méfiance chez Benny Morris, dans Norman G. Finkelstein, Image and Reality of the IsraelPalestine Conflict, New York, Verso, 1995, p. 56.

33. Sharif Kanaana, Still on Vacation!, Jérusalem, SHAML, 2000, pp. 145-172.

34. Ibid., pp. 85-144, par exemple.

35. Ilan Pappé, art. cit., p. 56.

36. Voir Elie Barnavi, « Calomniez, calomniez... », dans Le Monde du 4 juin 2002 ; et la réponse d'Ilan Pappé : « Telle est l'Affaire Katz », dans Le Monde du 5 juin ; ainsi que la pétition internationale, Petition for the Defense of Academic Freedom in Israel.

37. Elias Sanbar, art. cit., p. 124.

38. Sur les manuels scolaires, voir Nathan J. Brown, Democracy, History, and the Contest over the Palestinian Curriculum, conférence à l'Adam Institute, novembre 2001 ; Maureen Meehan, « Israeli Textbooks and Children's Literature Promote Racism and Hatred Toward Palestinians and Arabs ", dans Washington Report, septembre 1999, pp. 19-20; et Fuad Mughrabi, «Les manuels scolaires palestiniens sont-ils antisémites ? » dans la Revue d'études palestiniennes, $\mathrm{n}^{\circ}$ 82, hiver 2002, pp. 53-64.

39. Voir par exemple, à la jonction de la philosophie, de l'histoire et des sciences politiques, les actes du colloque rassemblés dans Tommis Kapitan, Philosophical Perspectives on the Israeli-Palestinian Conflict, s. 1., M. E. Sharpe, 1997. L'ouvrage est une collection de points de vue israéliens et palestiniens difficilement conciliables, reprenant les justifications traditionnelles des uns et des autres.

40. Henry Laurens, dans Esprit, été 2000, p. 144 (note).

41. Nûr Masalha, art. cit., pp. 16-17.

42. Uri Ram, « Mémoire et identité : sociologie du débat des historiens en Israël », dans Florence Heymann \& Michel Abitbol, L'Historiographie israélienne aujourd'hui, coll. 
" Mélanges du Centre de recherche français de Jérusalem », Paris, Éditions du CNRS, 1998, p. 200.

43. « 80 Theses for a New Peace Camp », dans Ha'aretz, 13 avril 2001.

\section{AUTEUR}

\section{PHILIPPE BOURMAUD}

Philippe Bourmaud est ancien élève de l'Ėcole normale supérieure et agrégé d'histoire. Il prépare une thèse sur les Transformations des conditions sanitaires et des pratiques de santé dans le sud du Bilâd al-Shâm, 1831-1918, sous la direction de Mme Randi Deguilhem à l'université de Provence et à l'Iremam. Il a été, d'octobre 2000 à septembre 2001, lecteur de français à l'université nationale An-Najah de Naplouse. pbourmaud(at)free.fr 